\title{
VALIDACIÓN DE GENOTIPOS DE FRIJOL NEGRO EN LA ZONA CENTRAL DEL ESTADO DE VERACRUZ, MEXICO ${ }^{1}$
}

\author{
Francisco Javier Ugalde Acosta ${ }^{2}$, Ernesto López Salinas ${ }^{2}$, Oscar Hugo Tosquy Valle ${ }^{2}$
}

\begin{abstract}
RESUMEN
Validación de genotipos de frijol negro en la zona central del estado de Veracruz, México. El objetivo del presente trabajo, fue validar genotipos de frijol con el paquete tecnológico del INIFAP, en parcelas de productores. Durante los ciclos de humedad residual de 1998 a 2001 se establecieron nueve parcelas de validación. Los genotipos utilizados fueron: las líneas II-307 y DOR-500 y las variedades Negro Huasteco 81, Negro Cotaxtla 91 y Negro INIFAP y como testigo del productor la variedad Jamapa. En las parcelas se realizaron las labores de manejo agronómico recomendadas por el INIFAP para esa zona de producción. Cada parcela ocupó una superficie total de $3000 \mathrm{~m}^{2}$. Se realizaron análisis de varianza individuales por localidad, considerando como repetición los rendimientos obtenidos por cada genotipo $\left(500 \mathrm{~m}^{2}\right)$ por ciclo de evaluación; también se efectuaron análisis combinado con prueba de separación de medias (DMS 5\%) y un análisis económico mediante la Tasa de Retorno Marginal. Los resultados indicaron que las variedades y líneas mejoradas fueron superiores en más del $100 \%$ en rendimiento al testigo. El análisis económico mostró que el mayor beneficio económico se obtuvo al sembrar las variedades Negro INIFAP y Negro Cotaxtla 91, con utilidades superiores a los US \$286/ha y una relación beneficio/costo de 1,52 y 1,51 , respectivamente.
\end{abstract}

Palabras claves: frijol, validación, adaptación a humedad residual, rendimiento.

\begin{abstract}
Validation of genotypes of black bean in the central area of the state of Veracruz, Mexico. The aims of this work was to validate bean growers' fields. During 1998 to 2001 residual moisture growing cycles, nine validation plots were established. The bean genotypes utilized were: the lines II-307 and DOR-500 and the varieties Negro Huasteco 81, Negro Cotaxtla 91, Negro INIFAP and Jamapa, which is traditionally sown by beans growers. All the materials were handled with the agronomical recommendations of INIFAP for that bean growing area. Each plot had an area of $3000 \mathrm{~m}^{2}$. Individual analyses of variance by locality were performed. The yields obtained for each genotype $\left(500 \mathrm{~m}^{2}\right)$ in one evaluation cycle were considered as replications. Combined analyses using the Marginal Return Rate were also performed. The results indicated that the yields of the varieties and improved lines were $100 \%$ higher than the yield of the traditional variety. The economic analysis showed that the highest economic profit was obtained when varieties Negro INIFAP and Negro Cotaxtla 91 were sown, being their profits higher than $\$ 3000 /$ ha and having a profit/cost relationship of 1.52 and 1.51 , respectively.
\end{abstract}

Key words: bean, validation, adaptation to residual moisture, yield.

\section{INTRODUCCIÓN}

En el estado de Veracruz la superficie promedio cosechada de frijol negro en el período de 1996-2000 fue de 39.507 ha con una producción de 24.987 toneladas y un rendimiento medio de $638 \mathrm{~kg} / \mathrm{ha}$, sin embargo, las estadísticas reflejan una tendencia a la baja en la superficie de frijol de 18,2\% (SAGARPA 2001). La zona central de la entidad participa con una superficie de dos mil hectáreas sembradas en un $98 \%$ en el ciclo otoñoinvierno de humedad residual, donde se obtiene un rendimiento promedio de $409 \mathrm{~kg} / \mathrm{ha}$ (INEGI 2002). Una de las causas de este bajo rendimiento es la utilización ciclo con ciclo de grano de la variedad Jamapa, obtenido sin ningún proceso de selección y beneficio, lo cual no garantiza que se mantenga la pureza genética del

1 Recibido: 4 de octubre, 2004. Aceptado: 15 de diciembre, 2004. Presentado en la L Reunión Anual del PCCMCA, El Salvador, abril del 2004.

2 Campo Experimental Cotaxtla-INIFAP. Km. 34 carretera Veracruz- Córdoba. A.P. 429 Veracruz, Ver., México. Correo electrónico: ugalde.francisco@inifap.gob.mx y salinaser@hotmail.com 
cultivar, así como el uso de materiales criollos con características agronómicas indeseables y de limitada capacidad productiva (López et al. 1999 b). Aunado a lo anterior, los altos costos de producción han hecho menos rentable el cultivo, lo que ha provocado la reducción de la superficie de siembra.

El Programa de Investigación de Frijol del Campo Experimental Cotaxtla perteneciente al INIFAP, ha desarrollado en las últimas dos décadas, variedades mejoradas de frijol negro con amplia adaptación, alta capacidad productiva, y/o tolerantes a las principales enfermedades del trópico, a suelos de baja fertilidad y a la sequía en las etapas finales del cultivo (López y Acosta 1998). Como parte de este proceso, la validación de tecnología juega un papel importante, al verificar la relevancia de los resultados experimentales bajo condiciones de suelo, clima y manejo por parte de los agricultores ( Tosquy et al. 1995), y tiene como objetivo observar el comportamiento de los genotipos a nivel semicomercial, lo que permite cuantificar el incremento en la productividad, reacción a los factores adversos y aceptación comercial. En este sentido, Zuloaga et al. (1984) señala que la validación permite confirmar la hipótesis que la tecnología generada por el Campo Experimental supera en rendimiento, beneficio económico y/o en otras características a la tecnología que usan los productores.

En el periodo comprendido entre 1981-1998 fueron liberadas las siguientes variedades: Negro Veracruz, Negro Huasteco 81, Negro Cotaxtla 91, Negro INIFAP y Negro Tacaná (López et al. 1987; Yoshii et al. 1987; López y Rodríguez 1992; Villar y López 1993; López et al. 1996). Recientemente en el año 2002, fueron liberadas dos variedades Negro Tropical (DOR-500) y Negro Medellín (II-307) (López et al. 1999 a; López et al. 2000). El Programa de Frijol del Campo Experimental Cotaxtla validó en la zona central de Veracruz dos genotipos experimentales y tres variedades liberadas, con el objetivo de conocer su comportamiento en cuanto a rendimiento, adaptación a las condiciones edafoclimáticas y manejo por parte del productor, y de esta manera definir genotipos alternativos que permitan elevar la productividad y con ello incentivar la siembra del cultivo de frijol.

\section{MATERIALES Y MÉTODOS}

Durante los ciclos agrícolas de humedad residual otoño-invierno 1998-99, 1999-00 y 2000-01 se establecieron en total nueve parcelas de validación de genotipos en terrenos de productores; tres en Jamapa, tres en Medellín de Bravo y tres en Veracruz, ubicadas en la zona central del estado de Veracruz. Las características de suelo y clima se presentan en el Cuadro 1.

Cada parcela constó de cinco genotipos y un testigo tradicional y ocupó una superficie total de $3000 \mathrm{~m}^{2}$. Los genotipos sembrados fueron: las líneas experimentales II-307 y DOR-500 y variedades Negro Huasteco 81, Negro Cotaxtla-91, Negro INIFAP y como testigo tradicional se utilizó la variedad Jamapa sembrada por el productor.

Las siembras de las parcelas se realizó durante el mes de septiembre. En las parcelas con suelos franco y migajón-arenoso se depositaron 15 semillas por metro líneal en surcos separados a $60 \mathrm{~cm}$, y en suelos arenosos se depositaron tres semillas cada $30 \mathrm{~cm}$ en surcos separados a $40 \mathrm{~cm}$; para ambos métodos de siembra la densidad fue de 250,000 plantas/ha. La fórmula de fertilización empleada fue la 40-40-0, para lo cual se utilizó la mezcla física con las fuentes nutrimentales urea y fosfato diamónico con grado 18-46-0 aplicada en una sola banda al lado del surco en las localidades establecidas en suelos francos, y mateada en las localidades con suelos arenosos a los ocho días después de la emergencia. El control de malezas se realizó en forma manual dentro de los primeros 30 días de nacido el cultivo. Debido a la presencia de doradilla (Diabrótica balteata Le Conte) y chicharrita (Empoasca kraemeri) en la etapa vegetativa V1 se aplicó Cipermetrina $24 \%$ en dosis de $1 \mathrm{ml} / \mathrm{l}$ de agua. La cosecha de los genotipos en cada

Cuadro 1. Características edafoclimáticas de las localidades donde se establecieron las parcelas de validación de frijol. Veracruz, México. 1998-2001.

\begin{tabular}{lcccccc}
\hline \multicolumn{1}{c}{ Localidad } & $\begin{array}{c}\text { Altitud } \\
(\mathbf{m})\end{array}$ & $\begin{array}{c}\text { Temperatura } \\
\text { media anual }(\mathbf{C})\end{array}$ & $\begin{array}{c}\text { Precipitación } \\
\text { anual }(\mathbf{m m})\end{array}$ & $\begin{array}{c}\text { Tipo de } \\
\text { clima }\end{array}$ & $\begin{array}{c}\text { Textura de } \\
\text { suelo }\end{array}$ & pH \\
\hline Jamapa,Ver. & 18 & 25 & 1.620 & $\mathrm{AW}_{2}$ & Franco & 6,7 \\
Medellín de & & & & & & \\
Bravo, Ver. & 16 & 25 & 1.484 & $\mathrm{AW}_{1}$ & Migajón-arenoso & 6,3 \\
Santa Rita, Ver. & 4 & 26 & 1.054 & $\mathrm{AW}_{0}$ & Arenoso & 5,1 \\
\hline
\end{tabular}


parcela se llevó a cabo a los 90 días después de la siembra. El peso de campo obtenido en el área total de cada genotipo se transformó en rendimiento de grano en kilogramos por hectárea, ajustado al 14\% de humedad.

Se realizaron análisis de varianza individuales por localidad, considerando como repeticiones los rendimientos obtenidos de cada genotipo (que ocupó una superficie de $500 \mathrm{~m}^{2}$ ) por ciclo de evaluación y análisis combinado para determinar la interacción localidad por genotipo. Como prueba de comparación de medias se aplicó la Diferencia Mínima Significativa (DMS) al 5\% de probabilidad de error. Además se realizó un análisis económico por localidad, para determinar la mejor opción tecnológica de variedades mejoradas en función de la Tasa de Retorno Marginal (CIMMYT 1988).

\section{RESULTADOS Y DISCUSIÓN}

Los rendimientos de grano obtenidos por los genotipos en cada año de evaluación en las localidades de Jamapa, Veracruz y Medellín de Bravo, se presentan en los Cuadros 2, 3 y 4, respectivamente, donde se puede apreciar que los más altos rendimientos se consiguieron durante el ciclo O-I 1998/99, debido a que se tuvo una mayor precipitación durante el ciclo $(614 \mathrm{~mm})$ y una mejor distribución de las lluvias en las etapas de floración, formación de vainas y llenado de grano (354,3 $\mathrm{mm}$ ). Para el caso de los otros dos ciclos homólogos, la precipitación total fue de 430 y $427 \mathrm{~mm}$ respectivamente, además se presentó una distribución irregular de las lluvias, con tan sólo $32 \mathrm{~mm}$ en el ciclo 1999/00 y 45 mm en el ciclo 2000/01 en las etapas arriba señaladas. Lo arriba señalado pone de manifiesto, que el factor ambiental tiene gran influencia en el rendimiento de los genotipos, tal como lo mencionan Stoker (1974) y

Cuadro 2. Rendimientos de grano ( $\mathrm{kg} / \mathrm{ha}$ ) obtenidos de parcelas de validación de genotipos de frijol sembradas en el ciclo O-I de 1998- 2001 bajo humedad residual en Jamapa. Veracruz, México. 1998-2001.

\begin{tabular}{lcrrrr}
\hline \multicolumn{1}{c}{ Variedad } & $\begin{array}{c}\text { 1998- } \\
\mathbf{9 9}\end{array}$ & $\begin{array}{r}\text { 1999- } \\
\mathbf{0 0}\end{array}$ & $\begin{array}{r}\mathbf{2 0 0 0 -} \\
\mathbf{0 1}\end{array}$ & $\begin{array}{r}\text { Promedio } \\
\text { relativo }\end{array}$ & $\begin{array}{c}\text { \% } \\
\text { DOR-500 }\end{array}$ \\
& 1.911 & 1.321 & 793 & $1.341,7$ & 350,8 \\
N. INIFAP & 1.947 & 1.005 & 991 & $1.314,3$ & 343,78 \\
N. Huasteco 81 & 2.103 & 1.112 & 700 & $1.305,0$ & 341,4 \\
N. Cotaxtla 91 & 1.547 & 750 & 1.240 & $1.179,0$ & 308,4 \\
II-307 & 1.478 & 1.019 & 870 & $1.122,3$ & 293,56 \\
Jamapa (T) & 597 & 400 & 150 & 382,3 & 100,0 \\
Promedio & $1.597,2$ & 934,5 & 790,7 & $1.107,4$ & \\
\hline
\end{tabular}

Cuadro 3. Rendimientos de grano (kg/ha) obtenidos de parcelas de validación de genotipos de frijol sembradas en el ciclo O-I de 1998- 2001 bajo humedad residual en Veracruz, Ver. México. 1998-2001.

\begin{tabular}{lccccc}
\hline Variedad & $\begin{array}{c}\text { 1998- } \\
\mathbf{9 9}\end{array}$ & $\begin{array}{c}\text { 1999- } \\
\mathbf{0 0}\end{array}$ & $\begin{array}{c}\text { 2000- } \\
\mathbf{0 1}\end{array}$ & $\begin{array}{c}\text { Promedio } \\
\text { relativo }\end{array}$ \\
\hline & & & & & \\
N. Cotaxtla & 1.450 & 714 & 1.540 & $1.234,7$ & 262,7 \\
II-307 & 1.478 & 810 & 940 & 1076 & 228,9 \\
DOR-500 & 1.584 & 654 & 908 & $1.048,7$ & 223,1 \\
N. INIFAP & 1.217 & 758 & 800 & 925 & 196,8 \\
N. Huasteco 81 & 966 & 675 & 700 & 780.3 & 166 \\
Jamapa (T) & 640 & 478 & 292 & 470 & 100 \\
Promedio & $1.222,5$ & 681,5 & 863,3 & 922,4 & \\
\hline
\end{tabular}

Cuadro 4. Rendimientos de grano (kg/ha) obtenidos de parcelas de validación de genotipos de frijol sembradas en el ciclo O-I del 1998- 2001 bajo humedad residual en Medellín de Bravo, Ver, México. 1998-2001.

\begin{tabular}{lrrrrr}
\hline Variedad & $\begin{array}{r}\text { 1998- } \\
\mathbf{9 9}\end{array}$ & $\begin{array}{r}\text { 1999- } \\
\mathbf{0 0}\end{array}$ & $\begin{array}{r}\mathbf{2 0 0 0 -} \\
\mathbf{0 1}\end{array}$ & $\begin{array}{r}\text { Promedio } \\
\text { relativo }\end{array}$ & $\begin{array}{l}\text { \% } \\
\text { N. INIFAP }\end{array}$ \\
& 1.798 & 1.131 & 1.118 & $1.349,0$ & 311,3 \\
N. Huasteco 81 & 1.595 & 1.200 & 1.120 & $1.305,0$ & 301,2 \\
N. Cotaxtla & 1.140 & 1.044 & 1.280 & $1.154,7$ & 266,5 \\
II-307 & 1.264 & 1.074 & 1.030 & $1.122,7$ & 259,1 \\
DOR-500 & 1.788 & 853 & 670 & $1.103,7$ & 254,7 \\
Jamapa (T) & 560 & 320 & 450 & 443,3 & 100 \\
Promedio & $1.357,5$ & 937,0 & 944,7 & $1.079,7$ & \\
\hline
\end{tabular}

Acosta y Koshashi (1989). Cabe destacar que en todos los ciclos de validación, el testigo Jamapa utilizado por el productor, obtuvo los más bajos rendimientos, los cuales reflejan el rendimiento medio de la región. Esto es debido a que generalmente la semilla utilizada por el productor de frijol, proviene de siembras comerciales de producción de grano y como consecuencia no lleva un proceso de producción de semilla, ni de tratamiento fitosanitario. Esto indica que el uso de semilla de variedades mejoradas es uno de los mejores medios disponibles para que en corto plazo se pueda incrementar la productividad y rentabilidad del cultivo de frijol de humedad residual, tal como lo señala Zuloaga et al. (1984) y como se muestra en los porcentajes relativos obtenidos por las variedades en comparación con el testigo tradicional. En este sentido, la semilla mejorada es uno de los insumos estratégicos clave, para lograr incrementos en la productividad y la elección de una buena semilla es el primer paso para aprovechar de forma óptima los recursos disponibles (Tosquy et al. 1995). 
Los análisis de varianza para la variable rendimiento de grano en cada una de las localidades de prueba detectaron diferencias altamente significativas para los tratamientos en las localidades de Jamapa y Medellín de Bravo y significativa para el sitio de Veracruz, lo cual indica el diferente comportamiento en la capacidad productiva de los genotipos, dado por la constitución genética de los mismos.

De acuerdo a la DMS (Cuadro 5), en las localidades de Jamapa y Medellín de Bravo, tanto las variedades comerciales como las líneas experimentales tuvieron un comportamiento similar y superior estadísticamente en rendimiento de grano al testigo regional. En Veracruz, aunque cuatro genotipos resultaron sobresalientes y estadísticamente similares entre sí, el mejor comportamiento productivo lo obtuvo la línea DOR-500. Este mismo comportamiento se ha observado en siembras establecidas en suelos arenosos de Isla,

Cuadro 5. Rendimientos de grano promedio obtenido por las variedades en cada localidad México. 1999-2001.

\begin{tabular}{|c|c|c|c|c|}
\hline No. & Variedad & $\begin{array}{c}\text { Jamapa } \\
\text { (kg/ha) }\end{array}$ & $\begin{array}{c}\text { Veracruz } \\
\text { (kg/ha) }\end{array}$ & $\begin{array}{c}\text { Medellín } \\
\text { (kg/ha) }\end{array}$ \\
\hline 1 & N. INIFAP & $1.314,3 \mathrm{a}^{1}$ & $1.076,0 \mathrm{ab}$ & $1.305,0 \mathrm{a}$ \\
\hline 2 & N. Cotaxtla 91 & $1.179 \mathrm{a}$ & $925,0 \mathrm{ab}$ & $1.122,7 \mathrm{a}$ \\
\hline 3 & DOR-500 & $1.341,7 \mathrm{a}$ & $1.234,7 \mathrm{a}$ & $1.349,0 \mathrm{a}$ \\
\hline 4 & N. Huasteco 81 & $1.305,0 \mathrm{a}$ & $1.048,7 \mathrm{ab}$ & $1.154,7 \mathrm{a}$ \\
\hline 5 & II-307 & $1.122,3 \mathrm{a}$ & 780,3 bc & $1.103,7 \mathrm{a}$ \\
\hline \multirow[t]{2}{*}{6} & T. Jamapa & $382,3 \quad b$ & $470,0 \quad c$ & $433,3 \quad b$ \\
\hline & DMS 0,05 & 470,9 & 388,3 & 424,5 \\
\hline
\end{tabular}

\footnotetext{
${ }^{1}$ Valores con las mismas letras no son diferentes estadísticamente.
}

Veracruz, México, donde está misma línea ha mostrado buena respuesta (López et al. 1999).

En el análisis combinado sólo se detectó significancia en el factor variedades, lo que indica que existieron diferencias en la capacidad productiva de los genotipos. La no significancia encontrada en el factor Localidades y en la interacción Localidades x Tratamientos obedeció principalmente en el primer caso, a que las condiciones climáticas fueron muy similares en las tres localidades. Los rendimientos en estas localidades fueron de $1.107,1.079$ y $922 \mathrm{~kg} / \mathrm{ha}$ para Jamapa, Medellín y Veracruz. Esta última localidad obtuvo el menor rendimiento debido a su textura arenosa, las plantas en general, fueron sometidas a un mayor estrés hídrico en las etapas de formación de vainas y llenado de grano. La no significancia detectada en la acción conjunta L x V señala que las variedades se comportaron de manera similar de un ambiente de evaluación a otro.

El rendimiento promedio obtenido por los genotipos de frijol a través de las localidades de evaluación se presentan en el Cuadro 6, donde se puede apreciar que tanto las variedades liberadas como las líneas experimentales evaluadas superaron ampliamente al testigo regional, lo cual pone de manifiesto la superioridad en el potencial productivo que presentan estos genotipos. El análisis económico mostró que el mayor beneficio económico se obtuvo al sembrar las variedades Negro INIFAP y Negro Cotaxtla 91, con las cuales se obtuvieron utilidades superiores a US \$ 286 por hectárea y las mejores relaciones beneficio costo con 1,52 y 1,51 , respectivamente. Los resultados muestran la importancia que tiene la validación de los genotipos de frijol al mostrar a los productores los beneficios de productividad y rentabilidad de las variedades mejoradas y su posible aceptación. Coincidiendo por lo expresado por Gittinger

Cuadro 6. Rendimiento promedio y análisis económico de genotipos de frijol. Veracruz, México1999-2001.

\begin{tabular}{llcccc}
\hline No. & Variedad & $\begin{array}{c}\text { Rendimiento } \\
(\mathbf{k g} / \mathbf{h a})\end{array}$ & $\begin{array}{c}\text { Ingreso bruto } \\
(\mathbf{U . S .} \text {. })^{\mathbf{1}}\end{array}$ & $\begin{array}{c}\text { Beneficio neto } \\
\text { (U.S. } \mathbf{~})\end{array}$ & Rel. B/C \\
\hline & & & & & \\
4 & N. INIFAP & $1.196,1$ & 852,8 & 290,8 & 1,52 \\
1 & N. Cotaxtla & $1.189,4$ & 848,0 & 286,0 & 1,51 \\
2 & DOR-500 & $1.130,1$ & 805,8 & 243,8 & 1,43 \\
5 & N. Huasteco 81 & $1.130,1$ & 805,8 & 243,8 & 1,43 \\
3 & II-307 & $1.107,0$ & 789,3 & 227,3 & 1,40 \\
6 & T. Jamapa & 431,9 & 307,9 & $-254,1$ & 0,55 \\
\hline
\end{tabular}

${ }^{1}$ U.S. $\$=$ a $\$ 11.21$ pesos mexicanos. Costo producción aplicado por el productor U.S \$ 562.00. Precio de venta por kilogramo de grano U.S \$ 0.713. 
(1973), al mencionar que resultados con una relación beneficio-costo igual uno o superior a uno, dan confiabilidad a las recomendaciones de siembras de nuevas variedades en terrenos de productores.

Con base en los resultados obtenidos se concluye que tanto las líneas experimentales como las variedades mejoradas superaron significativamente en rendimiento al testigo regional. Las variedades Negro INIFAP y Negro Cotaxtla 91 representaron las mejores alternativas tecnológicas y económicas para los productores de frijol de humedad residual en los municipios de Jamapa, Veracruz y Medellín de Bravo de la zona central del estado de Veracruz.

\section{LITERATURA CITADA}

ACOSTA, J.A.; KOSHASHI S. 1989. Effect of water stress on growth and yield of in determinate dry bean (Phaseolus vulgaris) cultivars. Field Crops Res 20:81-93

CIMMYT (Centro Internacional para el Mejoramiento de Maíz y Trigo). 1988. La formulación de recomendaciones a partir de datos agronómicos. Un manual metodológico de evaluación económica. K. Cassaday y A. McNab (eds.). México, D.F. 79 p.

GITTINGER, J.P. 1973. Análisis económicos de proyectos agrícolas. Editorial Tecnos. Madrid. España. p. 12-119.

INEGI (Instituto Nacional de Estadística, Geografía e Informática). 2002. Anuario estadístico del estado de Veracruz. Tomo II. Aguascalientes, Ags., México. p. 638.

LÓPEZ, E.; ACOSTA, J.A. 1998. Mejoramiento de frijol negro mesoamericano en el trópico de México In: Lépiz, R. (ed). Memoria Taller Internacional de Mejoramiento Genético de Frijol Negro Mesoamericano. Veracruz, Ver., México. p.15-16.

LÓPEZ, E.; RODRÍGUEZ, R. 1992. Negro Cotaxtla 91, nueva variedad de frijol para las zonas tropicales de Veracruz. Folleto Técnico No. 1. CECOT.CIRGOC. INIFAP. Veracruz, Ver., México. 8 p.

LÓPEZ, E.;BECERRA, E.N.; CANO, O.;ORTEGA, D.A.; ACOSTA, J.A. 1996. Adaptación y calidad tecnológica de la variedad Negro Tacaná. Agronomía Mesoamericana $7(1): 26-34$.
LÓPEZ, E.; YOSHII, K.; CERVANTES, M. 1987. Negro Veracruz, nueva variedad de frijol para las zonas central y sur de Veracruz. Fitotecnia Mexicana 9: 49-56

LÓPEZ, E.; ACOSTA, J. A. ;CANO, O.; FRAIRE, G.; BECERRA, E. N.; VILLAR, B.; CUMPIAN, J.,UGALDE, F. J. 1999 a. DOR-500 y II-307, dos líneas promisorias de frijol negro para el sureste de México. In: Barradas, H. (ed). Memoria XII Reunión CientíficaTecnológica Veracruz. Veracruz, Ver., México. p. 2023.

LÓPEZ, E.; ACOSTA, J. A.;CANO, O.; FRAIRE, G.; CUMPÍAN, J.; BECERRA, E. N.; VILLAR, B.; UGALDE, F. J.1999 b. Estabilidad de rendimiento de la línea de frijol negro DOR-500 en el trópico húmedo de México. Agronomía Mesoamericana 10(2): 69-74.

LÓPEZ, E.; CUMPÍAN, J.; BECERRA, E. N.; VILLAR, B.; UGALDE, F. J.; ACOSTA, J. A. 2000. Adaptación y rendimiento de la variedad de frijol Negro Medellín en el sureste de México. Agronomía Mesoamericana 11(2):47-52.

SAGARPA (Secretaría de Agricultura, Ganadería, Desarrollo Rural, Pesca y Alimentación). 2001. Anuario estadístico de la producción agrícola por cultivo. Centro de Estadística Agropecuaria. México, D.F.

STOKER, R. 1974. Effect on dwart beans on water stress at different phases of growth. N.J. Exper. Agr.2: 13-15

TOSQUY, O.; SIERRA, M.; RODRÍGUEZ, F.; CASTILLO, ORTIZ, J.; TINOCO, A.; SANDOVAL, A.; URIBE, S.1995. Validación del híbrido de maíz (Zea mays L.) de cruza doble H-512 en el estado de Veracruz, México. Agronomía Mesoamericana 6: 93-97.

VILLAR, B.; LÓPEZ, E.; 1993. Negro INIFAP, nueva variedad de frijol para Chiapas y regiones tropicales similares. Fitotecnia Mexicana 16(2):208-209.

YOSHII, K.; RODRÍGUEZ, J.; CARRIZALES, N.; IBARRA, J.F.; PÉREZ, P. 1987. Rendimiento y adaptación de la línea D- 145 tolerante al mosaico dorado del fríjol en el Sureste de México. Fitotecnia Mexicana 9: 57-63.

ZULOAGA, A.; DÍAZ, S.; GONZÁLEZ, H.; MENDOZA, S. 1984. Manual para parcelas demostrativas. Dirección General de Distritos y Unidades de Temporal. INIA, FIRCO. México, D.F. 66 p. 